\title{
Parosteal ossifying lipoma of the clavicle: A case report
}

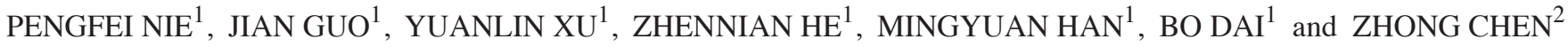 \\ ${ }^{1}$ Department of Orthopedics, Ningbo Beilun People's Hospital, Ningbo, Zhejiang 315800; \\ ${ }^{2}$ The First Affiliated Hospital of Zhejiang University School of Medicine, Hangzhou, Zhejiang 310000, P.R. China
}

Received October 15, 2015; Accepted March 21, 2016

DOI: $10.3892 / \mathrm{mco} .2017 .1135$

\begin{abstract}
Lipomas are very common benign adipose tissue tumors that most often develop in subcutaneous tissue. A limited number of studies have described the characteristics of parosteal ossifying lipomas located in the fibula, cervical vertebrae and mandible. However, to the best of our knowledge, parosteal ossifying lipoma of the clavicle has not yet been reported. We herein describe the clinical symptoms, radiological and histological findings in a rare case of parosteal ossifying lipoma of the clavicle in a 40-year-old male patient. The characteristic histological appearance, together with computed tomography and/or magnetic resonance imaging characteristics, should aid in the accurate diagnosis of such cases.
\end{abstract}

\section{Introduction}

Lipomas are very common benign lipomatous tumors that most often grow in the subcutaneous tissue. The majority of lipomas occur in adults aged $>30$ years, with an equal gender incidence. However, ossifying lipomas are rare. They have been reported at several sites in the body, including the soft tissues of the head and neck, the oral cavity, and the femur and fibular regions (1-6). Nitima et al were the first to report a case of parosteal ossifying lipoma occurring in the fibula of a 35-year-old female patient (6). To the best of our knowledge, parosteal ossifying lipomas of the clavicle have not been reported to date.

Parosteal ossifying lipomas develop over an extended period of time and present no clinical symptoms, except when they compress nerves. The clinical symptoms of osteochondromas are similar to those of parosteal ossifying lipomas, so the two types of tumors may be easily confused. We herein present our experience with the diagnosis and management of an ossifying lipoma adjacent to the periosteum of the clavicle.

Correspondence to: Dr Pengfei Nie, Department of Orthopedics, Ningbo Beilun People's Hospital, 1288 Lushan Road, Ningbo, Zhejiang 315800, P.R. China

E-mail: niepengfei188@163.com

Key words: parosteal ossifying lipoma, clavicle, lipoma, pathology

\section{Case report}

A 40-year-old man was admitted to Beilun People's Hospital due to a mass on his right clavicle, which had grown slowly over a period of 20 years. The mass insidiously increased in size, with no associated pain. The patient did not feel discomfort when he moved his neck. The patient's general health was good and there was no other remarkable medical history.

On physical examination, a mass sized $\sim 3 \times 2 \times 2 \mathrm{~cm}$ was palpable beneath the skin of the right shoulder and extending to the right clavicle. The mass was soft, non-tender and fixed to the clavicle. No prominent swelling, hyperaemia or ulceration of the skin were observed. The sensory and motor function of the right upper limb was normal. Preoperative examination revealed that the mass was well circumscribed peripherally and not adherent to adjacent muscles; however, it was fixed to the clavicle.

Radiographic examination revealed that the mass was located in the middle of clavicle, and its base intensity was similar to that of the clavicle; thus, the mass was initially suspected to be an osteochondroma (Fig. 1). On magnetic resonance imaging (MRI), the mass consisted of 3 layers (Fig. 2): The surface was encased in adipose tissue and the middle layer was osseous tissue; the base was fixed to the clavicle, but was not associated with clavicular marrow cavity. On T1- and T2-weighted images, the surface adipose tissue exhibited a high signal, while the core bone tissue exhibited a low signal.

Surgical treatment. En bloc resection of the mass together with the osseous protuberance of the right clavicle was performed. The mass was well circumscribed and was easily dissected from the adjacent soft tissue. The base of the tumour adhered strongly to the clavicle. The mass measured $3 \times 2 \times 2 \mathrm{~cm}$, with a smooth surface. The cut surface of the specimen was yellowish, with a mostly homogeneous appearance. The hard bony protuberance was chiselled from the clavicle; the mass was not continuous with the marrow cavity of the clavicle.

Histological examination. Microscopically, the mass was mostly composed of mature adipocytes, whereas mature chondrocytes were identified in the basal layer adherent to the clavicle (Fig. 3A). This mass consisted of three layers, namely an ossification center, bone tissue and adipose tissue. The ossification center resembled bone marrow, consisting of numerous osteogenic cells. The bone tissue included osteoblasts and chondrocytes surrounded by cartilage matrix. The 


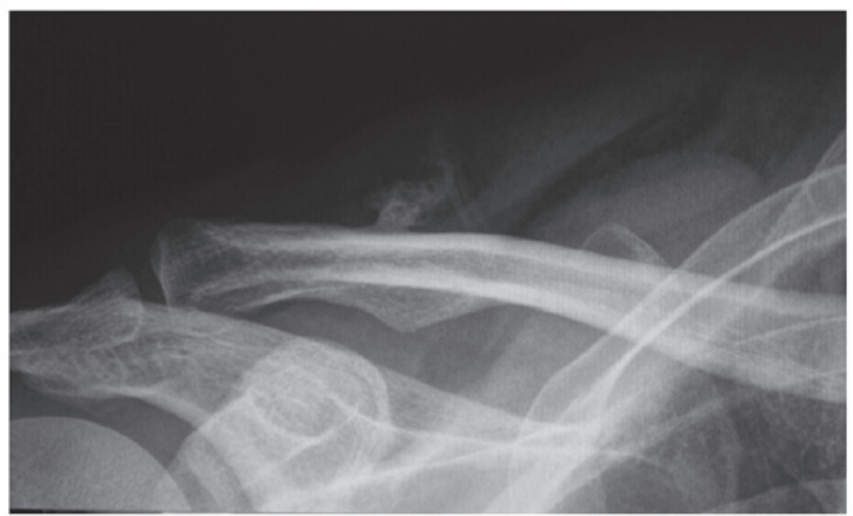

Figure 1. Plain radiograph of the right clavicle (front view) showing a well-defined radiolucent mass adjacent to the lateral aspect of the right clavicle. An irregularly-shaped, smoothly marginated osseous excrescence is seen within the radiolucent mass, attached to the cortex of the clavicle. No bone destruction was detected.

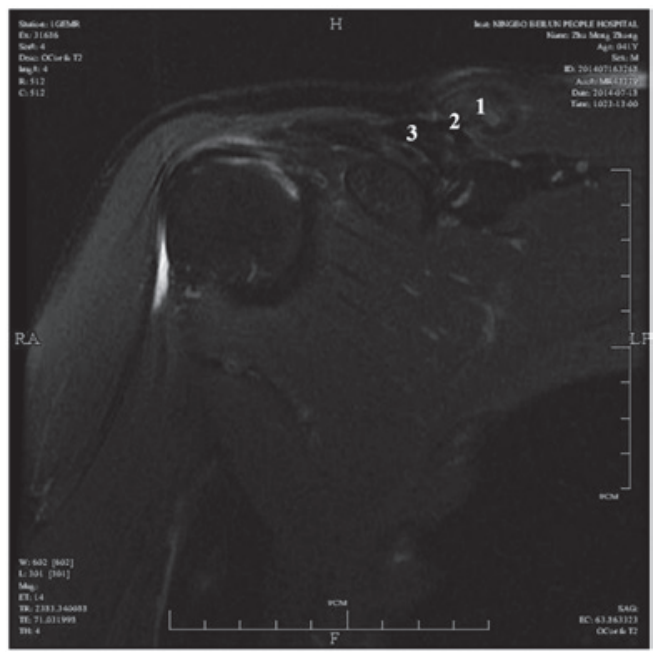

Figure 2. Coronal MR images showing a well-circumscribed soft tissue mass with low signal intensity on T2-weighted images. The mass was composed of a fat cap (1) and a large osseous base (2), and was adherent to the right clavicle (3).

adipose tissue was composed of mature adipose cells and fibrous tissue. None of the major components exhibited any nuclear pleomorphism or immaturity (Fig. 3B). Thus the diagnosis of ossifying parosteal lipoma was confirmed.

\section{Discussion}

Lipomas are the most common benign soft tissue neoplasms, accounting for $\sim 50 \%$ of all soft tissue neoplasms. Parosteal lipomas may be considered as a separate group of rare benign lipomas affecting the bones, performing either in mandible, cervical vertebra or fibula $(7,8)$. Parosteal lipomas are exceedingly rare benign lipomatous neoplasms located adjacent to the periosteum of bones. Parosteal lipomas have been reported at several sites in the body, including the soft tissues of the head and neck, the oral cavity, and the femur and fibular regions $(1-5,9)$. The most common site of parosteal lipomas is in the thigh, contiguous with the femur (6). To the best of our knowledge, parosteal lipomas in the clavicle have not been reported to date.
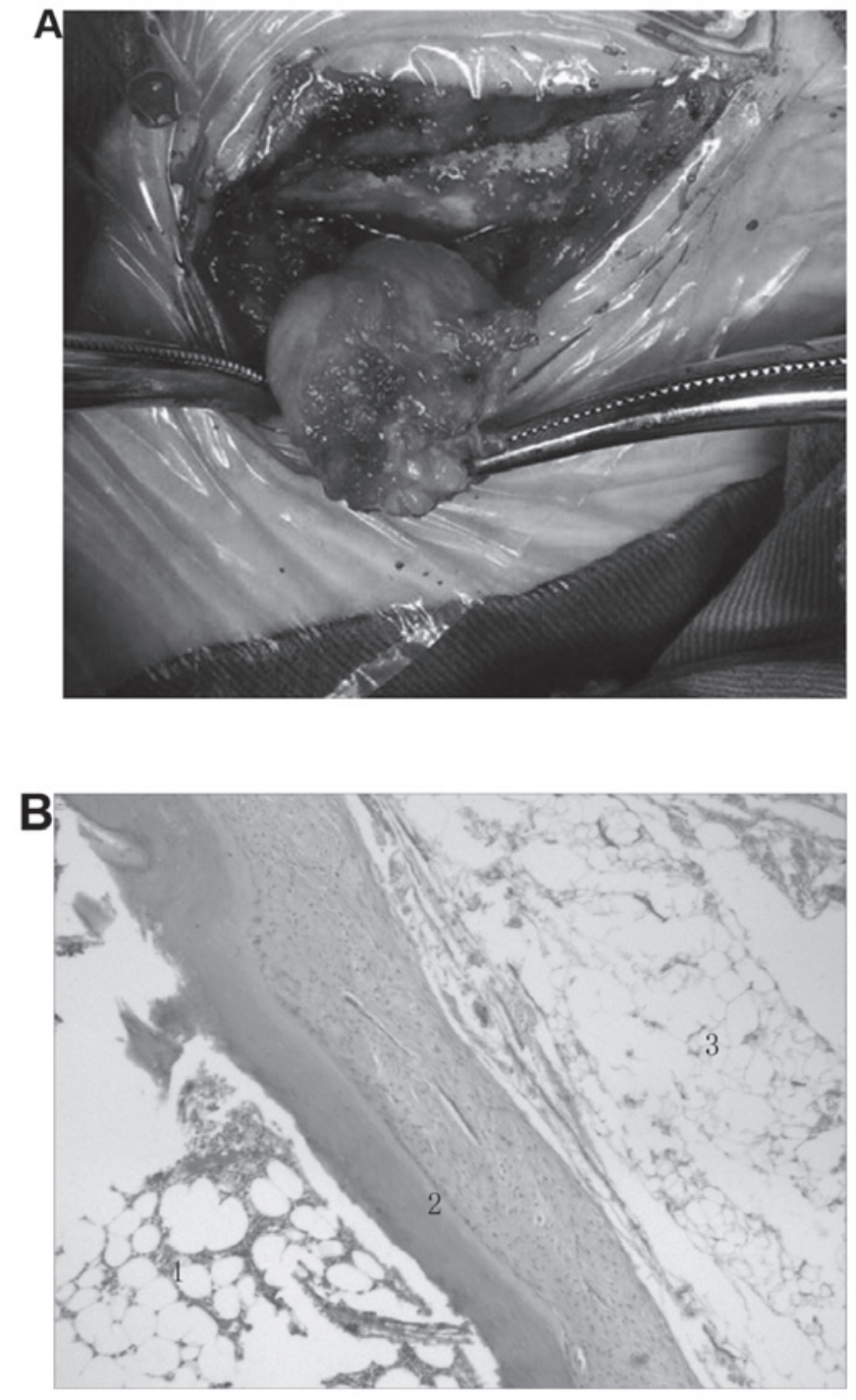

Figure 3. (A) Macroscopic appearance of the gross specimen. The tumor was a well-circumscribed spherical glistening yellowish mass, attached to the lateral aspect of the right clavicle. (B) Photomicrograph of the specimen showing the ossification center (1), bone tissue (2) and fat cap (3). The ossification center contains osteogenic progenitor cells. Bone tissue may be seen, containing active osteoblasts and chondrocytes (haematoxylin and eosin staining; magnification, $\mathrm{x} 40$ ).

Patients with parosteal lipomas are generally aged 40-60 years and they usually present with a slowly growing, painless mass fixed to the bone. Neurological deficits have occasionally been reported, most commonly associated with forearm lesions adjacent to the radius, resulting in posterior interosseous nerve palsy $(10,11)$. In the present case, the patient did not present with nerve palsy, paresthesia, or pain and the serum tumor marker levels were not elevated. This parosteal lipoma had been growing slowly over a period of 20 years and the patient wished to have the mass removed for aesthetic reasons alone.

Parosteal lipoma may occasionally be misdiagnosed as osteochondroma, when there is association with the underlying bone (12). In this case, the X-ray examination raised the suspicion of osteochondroma. On MRI, the mass was characterized by high signal intensity on T1-weighted images, with 
low signal of the bone layer. The mass exhibited low signal intensity on T2-weighted images (Fig. 2).

Intraoperatively, the mass was found to be wrapped with a pseudomembrane, had a large base and was fixed to the right clavicle. The surface of the mass was covered with a cap that was initially considered to be a cartilaginous cap. From the radiographic, MRI and macroscopic findings, the mass was highly suspected to be an osteochondroma; however, the pathological examination revealed that it was a parosteal ossifying lipoma (Fig. 3B). The mass did not have a cartilaginous cap, but consisted of an ossification center, bone tissue and adipose tissue. The cap covering the mass was composed of adipose tissue. The absence of a cartilaginous cap is a distinguishing characteristic between osteochondromas and ossifying lipomas.

The treatment of parosteal ossifying lipomas of the clavicle is complete surgical resection. Local recurrence is rare and malignant changes have not been documented to date. In this case, the periosteal lipoma was surgically removed by simple excision and there has been no evidence of recurrence.

To the best of our knowledge, this is the first reported case of parosteal ossifying lipoma of clavicle. The lesion was initially mistaken for osteochondroma. However, the cytohistomorphology of this case and the radiological characteristics supported the diagnosis of parosteal ossifying lipoma.

In conclusion, the clinical presentation of parosteal ossifying lipoma is similar to that of osteochondroma. The treatment in this case was complete surgical resection and the patient has experienced no recurrence, malignant transformation or metastasis.

\section{References}

1. Bognár L, Bálint K and Bárdóczy Z: Symptomatic osteolipoma of the tuber cinereum. Case report. J Neurosurg 96: 361-363, 2002.

2. Mackenzie IR, Girvin JP and Lee D: Symptomatic osteolipoma of the tuber cinereum. Clin Neuropathol 15: 60-62, 1996.

3. Moschopulos M, Becheanu G and Stamm B: Hypothalamic osteolipoma of the tuber cinereum. J Cell Mol Med 10: 240-242, 2006.

4. Hashmi AA, Malik B, Edhi MM, Faridi N and Ashraful M: A large parosteal ossifying lipoma of lower limb encircling the femur. Int Arch Med 7: 5, 2014

5. Diom ES, Ndiaye IC, Ndiaye M, Thiam A, Tall A, Nao EE, Diallo BK, Diouf R and Diop EM: Osteolipoma: An unusual tumor of the parotid region. Eur Ann Otorhinolaryngol Head Neck Dis 128: 34-36, 2011.

6. Saksobhavivat N, Jaovisidha S, Sirikulchayanonta V and Nartthanarung A: Parosteal ossifying lipoma of the fibula: A case report with contrast-enhanced MR study and a review of the literature. Singapore Med J 53: e172-e175, 2012.

7. Ramos A, Castello J, Sartoris DJ, Greenway GD, Resnick D and Haghighi P: Osseous lipoma: CT appearance. Radiology 157: 615-619, 1985.

8. Demiralp B, Alderete JF, Kose O, Ozcan A, Cicek I and Basbozkurt M: Osteolipoma independent of bone tissue: A case report. Cases J 2: 8711, 2009.

9. Sun Z, Sun L, Zhang Z and Ma X: Ossifying parosteal lipoma of the mandible: A case report and review of the literature. Dentomaxillofac Radiol 42: 57852073, 2013.

10. Murphey MD, Carroll JF, Flemming DJ, Pope TL, Gannon FH and Kransdorf MJ: From the archives of the AFIP: Benign musculoskeletal lipomatous lesions. Radiographics 24: 1433-1466, 2004.

11. Kawashima A, Magid D, Fishman EK, Hruban RH and Ney DR: Parosteal ossifying lipoma: CT and MR findings. J Comput Assist Tomogr 17: 147-150, 1993.

12. Brones A, Mengshol S and Wilkinson CC: Ossifying lipoma of the cervical spine. J Neurosurg Pediatr 5: 283-284, 2010. 